\title{
Reconstruction of directed neuronal networks in a microfluidic device with asymmetric microchannels
}

\author{
Josquin Courte ${ }^{*, \dagger, 1}$, Renaud Renault ${ }^{\ddagger}$, Audric Jan*, ${ }^{*}$, Jean-Louis Viovy*, \\ Jean-Michel Peyrin ${ }^{\dagger}$, Catherine Villard ${ }^{*}, 1$ \\ *Physico-Chimie Curie, Université PSL, CNRS, Institut Pierre-Gilles de Gennes \\ pour la Microfluidique, Paris, France \\ ${ }^{\dagger}$ Sorbonne Université, Institut Biologie Paris-Seine, CNRS, Inserm, \\ Neuroscience Paris-Seine, Paris, France \\ ${ }^{\ddagger}$ Koniku, Berkeley, CA, United States \\ ${ }^{\S}$ CNRS, Inserm, Institut de Biologie de l'Ecole Normale Supérieure, Paris, France \\ ${ }^{1}$ Corresponding authors: e-mail address: josquin.courte@curie.fr; catherine.villard@curie.fr
}

\section{CHAPTER OUTLINE}

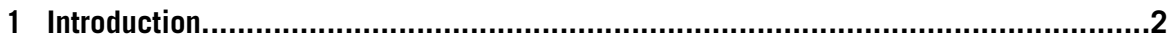

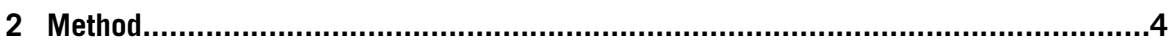

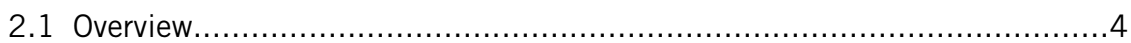

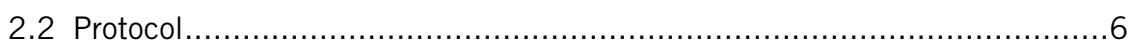

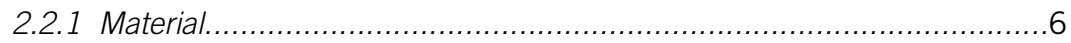

2.2.2 Fabrication of the PDMS Chip....................................................

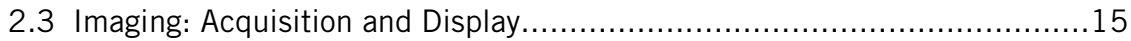

2.4 Automated Image Analysis...................................................... 15

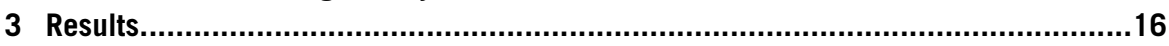

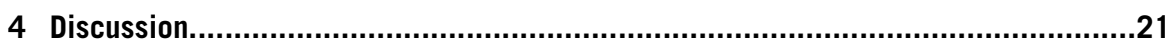

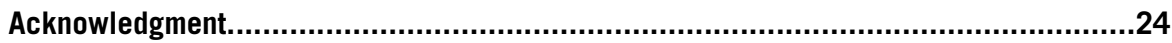

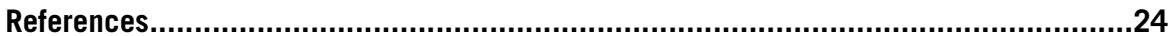

\section{Abstract}

Microfluidic devices for controlling neuronal connectivity in vitro are extremely useful tools for deciphering pathological and physiological processes occurring in neuronal networks. These devices allow the connection between different neuronal populations located into 
separate culture chambers through axon-selective microchannels. In order to implement specific features of brain connectivity such as directionality, it is necessary to control axonal growth orientation in these devices. Among the various strategies proposed to achieve this goal, one of the most promising and easily reproducible is the use of asymmetric microchannels. We present here a general protocol and several guidelines for the design, production and testing of a new paradigm of asymmetric microchannels geometries based on a "return to sender" strategy. In this method, axons are either allowed to travel between the emitting and receiving chambers within straight microchannels (forward direction), or are rerouted toward their initial location through curved microchannels (reverse direction). We introduce variations of these "arches" microchannels and evaluate their respective axonal filtering capacities. Importantly, one of these variants presents an almost complete filtration of axonal growth in the non-permissive direction while allowing robust axonal invasion in the other one, with a selectivity ratio as high as $99.7 \%$.

\section{INTRODUCTION}

Understanding normal and pathological brain function is an arduous task considering the intrinsic complexity of this organ. Neuronal processes are the result of the collective action of cells connected in a finely tuned, complex and dynamic 3D pattern. Physiological and pathological mechanisms thus have to be addressed in a network context. Gaining access to the connectome of in vivo neurons is very challenging, due to the abundance of both short- and long-range connections and to the multilayered architecture of the brain. Current experimental models used to study neurodegenerative diseases range from whole animal models that preserve the anatomical structures but greatly limit the experimentation at the cellular level to dissociated cell culture systems that allow detailed manipulation of cell phenotype but lack the highly ordered and instructive environment of the brain. These models are poorly adapted to efficiently and reliably study the detailed molecular/cellular phenomena at play. Thus, it is of prime interest to be able to reconstruct neuronal networks of controlled topologies in vitro and to manipulate each network node independently.

The development of tools for controlling neuronal connectivity in vitro has been the subject of much interest during the last decades. While seminal observations showed that distinct neuronal populations can form connections in vitro (Chubakov \& Sotnikov, 1982), exerting a fine control on this process has proven difficult. Based on the observation that neurons in culture requires specific adhesive molecules to attach and grow on a surface, microcontact printing and local protein/chemical deposition have been among the first strategies allowing to constrain neuronal outgrowth on flat substrates (Corey, Wheeler, \& Brewer, 1991; Wyart et al., 2002). Microfluidic techniques were later picked up as the next generation methods for controlling neuronal microenvironment. They mostly follow up on the seminal work of Campenot, who devised a method for fluidically isolating neurites from somas of cultured neurons with millimetric teflon inserts (Campenot, 1977). This technique was made more reproducible and accessible by Taylor and colleagues, thanks to the use of PDMS (polydimethylsiloxane) for 
fabricating chambers and rectangular microchannels (Taylor et al., 2005). It allows very efficient axon/soma compartmentalization of a great variety of neuronal subtypes and their fluidic isolation. In addition, neuronal populations introduced on both sides of these microchannels connect one to each other by generating a bidirectional network (Taylor, Dieterich, Ito, Kim, \& Schuman, 2010). However, directional connectivity being a fundamental feature of brain networks (Kale, Zalesky, \& Gollo, 2018), the possibility to orient axonal growth would increase the physiological relevance of these models. The first proof of concept that neuronal populations could be connected in a directional manner came from studies aiming at controlling axonal outgrowth through physical constraints using asymmetrical microchannels to guide axons (Peyrin et al., 2011). The use of asymmetric microchannels shapes has then been further extended (Gladkov et al., 2017; Le Feber, Postma, De Weerd, Weusthof, \& Rutten, 2015), and some of these designs allowed good performances regarding axonal filtration, reaching a selectivity ratio as high as 10 between the forward and reverse directions.

However, whereas these proofs of concept and their related performances have revealed useful for studying basic neurophysiological functions such as neurotransmission (Peyrin et al., 2011), diode-like electrical response (Renault et al., 2015) or pathological mechanisms (Deleglise et al., 2013, 2014; Virlogeux et al., 2018), the presence of some unwanted "retrograde" neuronal connections, even in very low proportion, can be problematic. This is particularly true for studying the trans-neuronal propagation of pathogenic, self-amplifying agents such as viruses and prion-like protein aggregates, particularly to evaluate the role of anterograde (from soma to the pre-synaptic compartment) versus retrograde (from post-synaptic compartment to soma) propagation (Volpicelli-Daley, Luk, \& Lee, 2014). Thus, it is of prime importance to dispose of a complete microfluidic toolbox including devices allowing $100 \%$ of axonal selectivity in mature networks.

We described in 2016 a new efficient paradigm for mechanical axonal filtration based on a "return to sender" strategy (Renault, Durand, Viovy, \& Villard, 2016). Our approach relies on two axonal pathfinding properties: (i) the tendency of axons to follow the corners defined by two perpendicular adhesive surfaces (here the edges of microchannels), and (ii) their finite flexural rigidity, defining a critical axonal bending angle. By using an array of straight and curved microchannels, we generate guiding edges or cusps depending on the axonal growth direction. Axons encountering cusps will ignore them and continue their path along the straight channel toward the opposite chamber. On the contrary, axons facing the convex side of curved microchannels will follow the curved edges and will be progressively guided back to their destination of origin. See Fig. 1 for an illustration of the principle of axonal filtration using arches.

Here, we describe in detail how to fabricate and handle microfluidic devices taking advantage of this paradigm for reconstructing oriented neuronal networks in vitro. In addition, we show variants of this design and evaluate their filtration power on axons originating from primary cultures of murine cortical and hippocampal neurons. Notably, one of these designs reaches $99.7 \%$ (cortical neuron) 


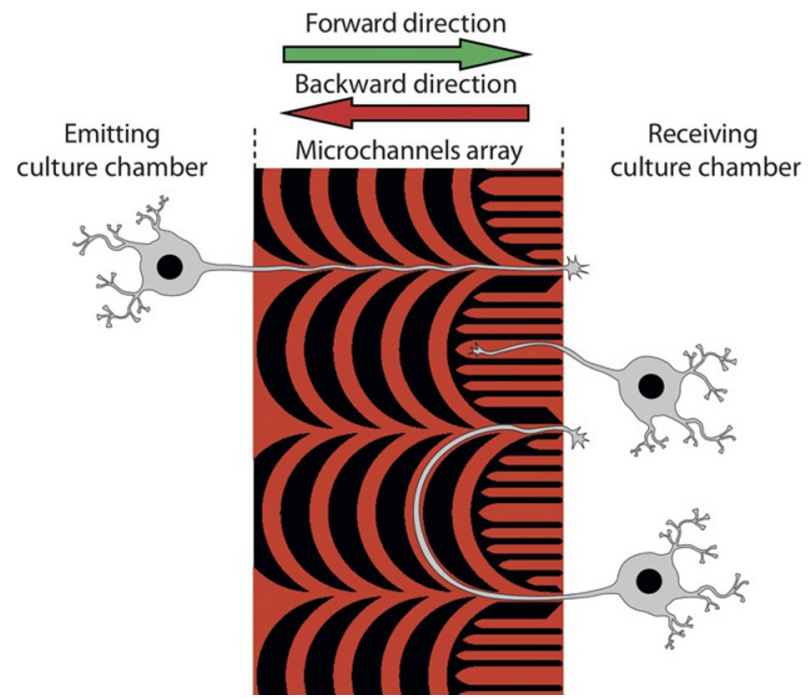

FIG. 1

Principle of axonal filtration by arches microchannels. Two culture chambers (blank spaces on left and right) are linked by an array of microchannels (in red). Axonal growth from the "emitting" culture chamber to the "receiving" culture chamber ("Forward direction") is unimpeded, with processes following a slightly undulating path. In contrast, axonal growth in the reverse direction ("Backward direction") is prevented by two strategies: (1) the implementation of "dead-end traps" to prevent or delay axonal growth inside the microchannels, and (2) the "return to sender" filtration supported by curved "arches" microchannels.

and $100 \%$ (hippocampal neurons) selectivity after 11 days of culture. Importantly, this orientation was preserved in a network context, where both chambers were seeded with neurons.

Different types of neuronal cultures may require specific geometric guiding clues. In order to allow members of the scientific community to develop new microchannels tailored to their needs, we present here a general protocol for microfluidic chips production from design to cell culture and guidelines for adequate microchannels design, and share a semi-automatized tool for quantifying axonal filtration. We also share the files encoding the culture chambers and directional microchannels, as well as the script allowing for semi-automated analysis of axonal transmission.

\section{METHOD}

\subsection{OVERVIEW}

This protocol covers every step from conception of microchannels shapes to cell seeding, with the exception of photomask fabrication, often subcontracted to specialized companies. The design of the photomask encoding microchannels and cell 
seeding chambers is made with a computer-assisted design (CAD) software. The resulting file (.dxf, .gerber, etc.) is used for photomask production. Fabrication of the initial mold is performed by photolithography. This mold can be used for the production of PDMS replicas, but is very brittle and can be damaged after several cycles of molding/demolding. To avoid too frequent time-consuming photolithography-assisted microfabrication, a sturdier resin mold can be generated from one or several PDMS replicas. Once demolded from the resin master, PDMS blocks containing the culture chambers and microchannels are bonded to glass coverslips using an oxygen or air plasma treatment. The resulting chips are sterilized, coated with adhesion molecules, and cells can be seeded in the desired compartments. The use of primary neuronal cultures expressing a fluorescent protein facilitates the dynamical observation of axonal growth without any staining protocol. After a few days of incubation, axonal growth can be assessed by fluorescence microscopy. Semi-automatic image processing allows the quantification of axonal outgrowth in both directions. In the following, the term "axonal chamber" will refer to the chamber opposite to the seeding chamber.

All devices described in this work contain two $1 \mathrm{~mm}$ wide culture chambers at both extremities of the microchannels, and a central chamber crossing this array (Fig. 2).

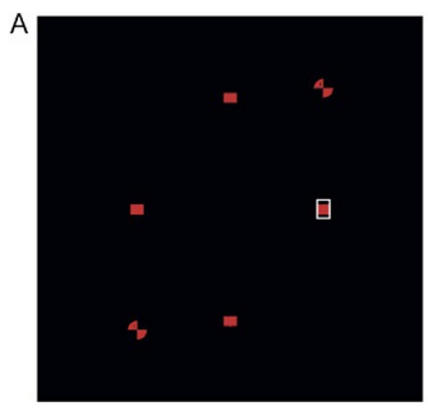

B

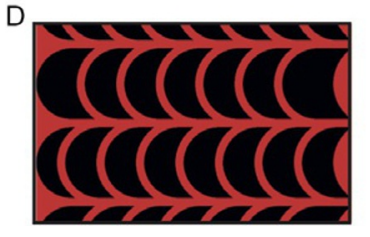

E
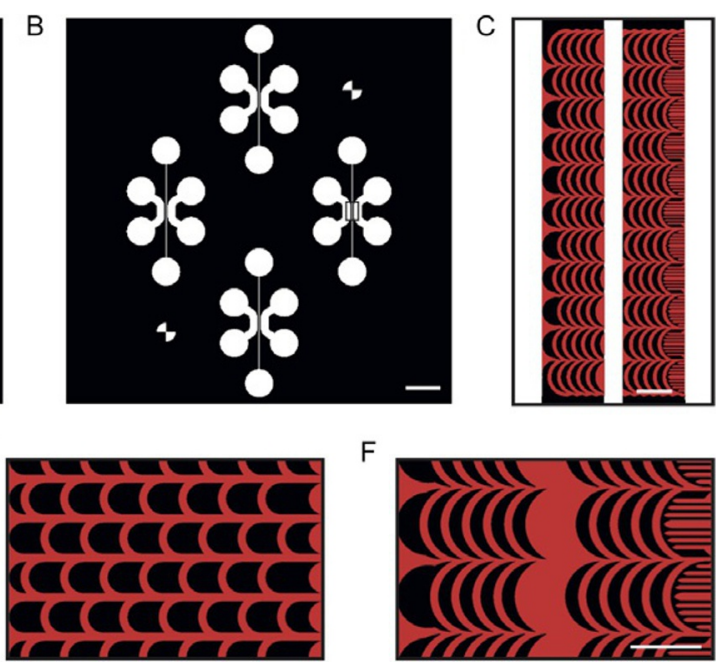

$F$

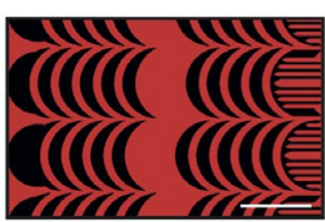

FIG. 2

Microchannels and culture chambers designs. (A) Culture chambers with central chamber (white) are designed with circles at both ends for easy wells punching. Four sets of chambers are designed for producing a chip containing four networks. Alignment crosses are present on both extremities of the design. (B) Microchannels (red) are designed with the same alignment crosses than the macrochambers. Scale bar for $A$ and $B$ is $5 \mathrm{~mm}$. (C) Zoom on the circled parts of A and B when both masks are combined: "sawtooth" arches microchannels (red) aligned with culture and central chambers (white). Scale bar is $100 \mu \mathrm{m}$. (D) $100 \mu \mathrm{m}$ diameter, $6 / 7$ repeats arches microchannels. (E) $40 \mu \mathrm{m}$ diameter, $6 / 7$ repeats arches microchannels. (F) "Sawtooth," 9 repeats arches microchannels with a space managed for not burying arches under central chamber. Scale bar for (D)-(F) is $100 \mu \mathrm{m}$. 
The central chamber has several functions: (1) to apply a counterflow during cell seeding, whatever one chamber or both are seeded. (2) to perform axotomy by briefly flowing a detergent solution in this chamber, as was performed in Deleglise et al. (2013). Moreover, this chamber allows for an efficient fluidic isolation of the culture chambers and thus the individual application of a separate treatment to each of them when this compartment is overpressurized (Fig. 3; see also Taylor et al., 2005). Lastly, "trap" patterns were designed to reduce the number of axons entering the microchannels in the reverse direction (see Fig. 1).

\subsection{PROTOCOL}

\subsubsection{Material}

\subsubsection{Reagents and consumables}

- Polydimethylsiloxane (base + curing agent) (PDMS, Ellsworth Adhesives)

- Glass coverslips, $5 \mathrm{~cm}$ diameter, 130-160 $\mu \mathrm{m}$ thickness (Fisher Scientific)

- Disposable $250 \mathrm{~mL}$ plastic cups (Dutscher)

- Square $10 \mathrm{~cm}$ wide plastic dish (Thermo Fisher Scientific)

- $6 \mathrm{~cm}$ petri dishes (Dutscher)

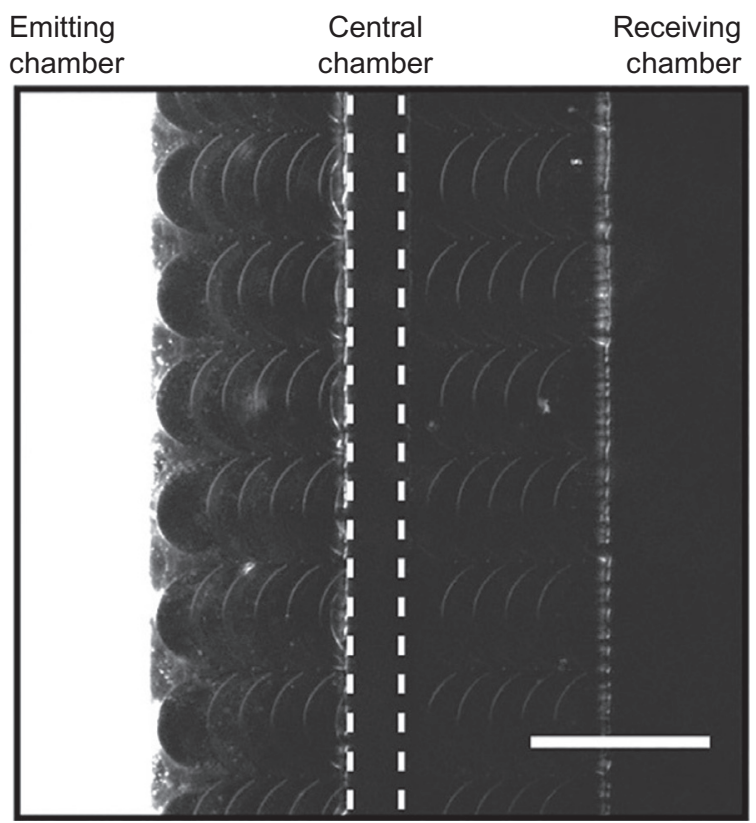

FIG. 3

Fluidical isolation in the device. A solution of Alexa-Fluor 488 coupled antibodies introduced in the emitting culture chamber does not flow to the receiving culture chamber during at least $24 \mathrm{~h}$ if a $100 \mu \mathrm{L}$ volume excess is maintained in the central chamber. Dotted lines define the central chamber. Scale bar is $100 \mu \mathrm{m}$. 
- Poly-D-lysine hydrobromide 30-70kDa (PDL) (Sigma)

- Laminin from Engelbreth-Holm-Swarm murine sarcoma (Sigma)

- SU8 negative photoresists 2002 and 2050 (MicroChem)

- SU8 photoresist developer: propylene glycol monomethyl ether acetate (PGMEA) (Technic)

- Sodalime chromium photomask with microchannels design (Selba)

- Plastic flexible photomask with culture chambers design (Selba)

- Isopropanol (VWR)

- Polyglass epoxy resin + reticulation agent (Esprit Composite)

- Primary murine neurons expressing fluorescent protein (Td Tomato)

- Cell culture medium

- $91 \%$ DMEM, High glucose, Glutamax supplement, pyruvate (Thermo Fisher Scientific)

- $5 \%$ Fetal Bovine Serum (GE Healthcare)

- $2 \%$ B-27 supplement (Thermo Fisher Scientific)

- $1 \%$ N-2 supplement (Thermo Fisher Scientific)

- $1 \%$ Penicillin/Streptomycin 10,000 U/mL (Thermo Fisher Scientific)

- Ethylenediaminetetraacetic acid disodium salt (EDTA) (Sigma)

- Dust-free wipes (Dutscher)

2.2.1.2 Equipment

- Clean room containing:

- MJB4 mask aligner (SUSS MicroTec)

- Spin-coater

- Hotplates $\left(65,95\right.$ and $\left.200^{\circ} \mathrm{C}\right)($ Stuart)

- Rotary shaker

- Fume hood

- Nitrogen spray gun

- Weighing scale

- $70^{\circ} \mathrm{C}$ oven (Memmert)

- Vacuum pump linked to desiccator (Ideal Vacuum)

- Oven dedicated to epoxy resin baking, placed under a fume hood (Memmert)

- Cell culture hood

- Plasma cleaner Pico PCCE (Diener electronic)

- UV sterilization cabinet

- Epifluorescence microscope (DMi8 from Leica, sCMOS Hamamatsu LT Flash 4.0 camera)

- Cell culture incubator $\left(37^{\circ} \mathrm{C}, 5 \% \mathrm{CO}_{2}\right.$, humidified atmosphere) (Panasonic)

\subsubsection{Fabrication of the PDMS chip}

\subsubsection{Rules for chips design}

Two photomasks have to be drawn, respectively, for microchannels and culture chambers, using for example open-source softwares (QCAD, Clewin, LibreCAD, etc.). Large culture chambers (Fig. 2A) can be fabricated from plastic photomasks 
while microchannels (Fig. 2B) typically require the micron-scale precision associated to "hard" chromium masks.

Several constraints resulting from the physical properties of the used materials (photoresist, PDMS) or from the rules governing axonal navigation might impact the proper fabrication and functioning of your device. These are to be kept in mind when designing your photomask and planning the height of the different structures.

- It is necessary to align properly microchannels with seeding chambers during photolithography. Draw at least a pairs of complementary alignment crosses on the two photomasks encoding microchannels and chambers. Placing them on both extremities of your masks allows a good angle-wise alignment. Making them big enough ( $2 \mathrm{~mm}$ or bigger) for preliminary alignment with naked eyes will speed up the alignment step (Fig. 2A and B).

- To ensure that microchannels will open on both chambers, an efficient method is to draw them longer than needed, so that their ends will be buried into the second (higher) layer of photoresist coding for the seeding chambers.

- Before drawing your photomasks, take note of the dimensions of the mask aligner holder. This will allow you to estimate the usable area of your mask.

- Long-term axonal health might be impacted by narrow microchannels $(<2 \mu \mathrm{m})$.

- The roof of your PDMS microchannels might collapse and stick to the bottom of the chip during post-plasma bonding. To avoid this, do not plan aspect ratios of $>1$ to 4 for channels (e.g., $3 \mu \mathrm{m}$ high, $12 \mu \mathrm{m}$ wide). If needed, add regularly interspaced micropillars for roof support $(12 \mu \mathrm{m}$ distant $2 \mu \mathrm{m}$ diameter pillars for $3 \mu \mathrm{m}$ high microchannels).

- To avoid the penetration of cell bodies into microchannels during cell seeding, microchannels should have a height comprised between 2 and $3 \mu \mathrm{m}$. Inducing a counterflow from the chamber opposite to the cell seeding chamber by filling it with medium before seeding the cells prevents the smallest of them to pass through. Cell migration (especially of glial cells) might still occur during culture time, albeit rarely.

- Avoid microchannels of excessive length that might impair axonal survival while delaying the formation of synaptic connections. Typical growth of axons through straight $500 \mu \mathrm{m}$ long microchannels occurs from DIV (day in vitro) 2 to DIV 4.

- Synchrony of neuronal activity between two neuronal populations requires a critical threshold of functional synaptic interconnectivity to be reached. Thus a minimal innervation degree, experimental conditions-dependent, has to be obtained. Having microchannels too far apart might prevent activity synchrony between the two populations.

- The outer walls of the channels located at both ends of the microchannels array are a potential source of defect for axonal selectivity by being able to guide axons in both directions. When designing a chip, one has to paid attention of coding several looped channels at the level of these outer walls to account for the absence of arches. 
- Very long (centimetric) macrochambers might generate heterogeneities in cell culture medium composition: areas next to the reservoirs will be preferentially perfused compared to the central ones, inducing gradients of nutriment with a risk of cell death in the center. Consequently, the length of the macrochambers (i.e., the distance between inlets) should be limited to millimetric dimensions.

\subsubsection{Master fabrication}

This protocol describes how to produce $3 \mu \mathrm{m}$ high microchannels linking $50 \mu \mathrm{m}$ high culture chambers on a 4 in. silicon wafer.

All of the following steps should be performed in a dust-free atmosphere. Use flat tweezers to manipulate silicon wafers.

\subsection{First layer (see Fig. 4A)}

1. Sprinkle a silicon wafer with isopropanol above an empty $80 \mathrm{~mm}$ diameter crystallizing dish ("trash") for about $5 \mathrm{~s}$. Place the wafer on an absorbent paper and dry it with pressurized nitrogen using an air blow gun.

2. Place the wafer on a hotplate for at least $3 \mathrm{~min}$ at $200^{\circ} \mathrm{C}$ to dry the surface, then transfer it on the bench to let it cool down to room temperature.

3. Place the wafer on a spin-coater. Check that the spin-coater is working properly by verifying that the aspiration of the wafer is effective (the wafer should spin and stay in place during the nominal rotation speed).

4. Gently pour a volume of SU8-2002 photoresist that covers about $70 \%$ of the wafer surface with a pipette. Eliminate eventual bubbles by aspiring them with the same pipette.

5. Spin-coat the photoresist on the wafer in two steps:

a. $500 \mathrm{rpm}$ for $10 \mathrm{~s}$ with acceleration of $100 \mathrm{rpm} / \mathrm{s}$

b. $700 \mathrm{rpm}$ for $30 \mathrm{~s}$ with acceleration of $300 \mathrm{rpm} / \mathrm{s}$

6. Soft bake: place the coated wafer on a first hotplate regulated at $65^{\circ} \mathrm{C}$ for $30 \mathrm{~s}$ then on a second one regulated at $95^{\circ} \mathrm{C}$ for $2 \mathrm{~min}$. Then transfer the wafer on the bench and let it cool down at room temperature.

7. Place the chromium mask onto the mask holder of the aligner, chrome side up, and turn on the vacuum to secure it. Flip the holder with the mask sucked onto it, chrome side down, and insert it into the aligner.

8. Place the coated wafer on the chuck of the mask aligner and set the distance between the mask and the wafer at $0 \mu \mathrm{m}$ ("Hard contact" mode).

9. Expose the first layer to UV light through the chromium mask. With a $3 \mu \mathrm{m}$ layer of SU8 photoresist, exposure dose should be about $90 \mathrm{~mJ} / \mathrm{cm}^{2}$. The exposure time is calculated by dividing the exposure energy $E$ specific for each photoresist by the effective power of the UV lamp, modulated by the absorption of the mask: $P_{\text {eff }}=P_{\text {lamp }}\left(1-\right.$ Absorption $\left._{\text {mask }}\right)$. Absorption is about $0.2(20 \%)$ for plastic masks and negligible for chromium mask.

10. Remove the wafer from the aligner and soft bake as in step 6. Let it cool down at room temperature. 
A

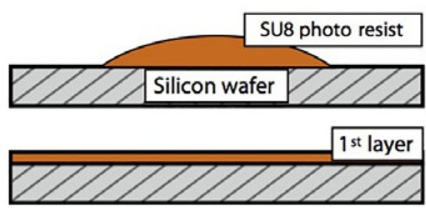

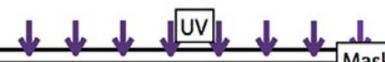

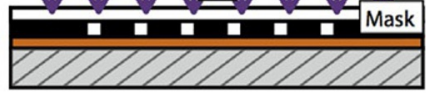

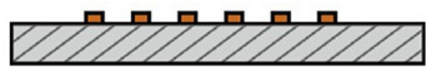

B
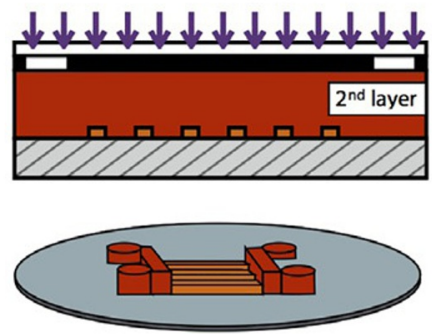

C
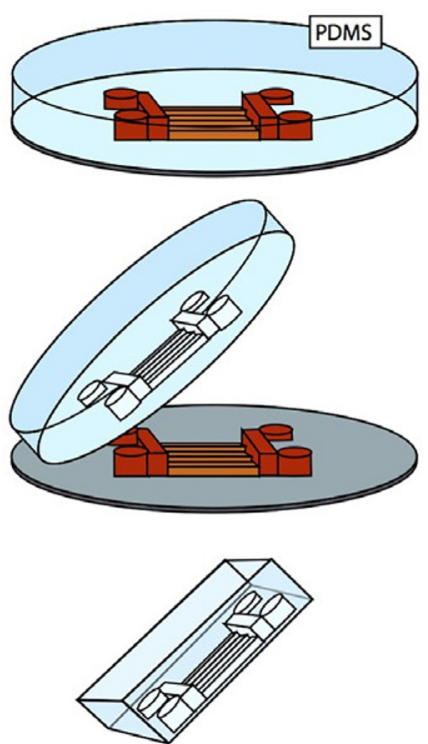

D
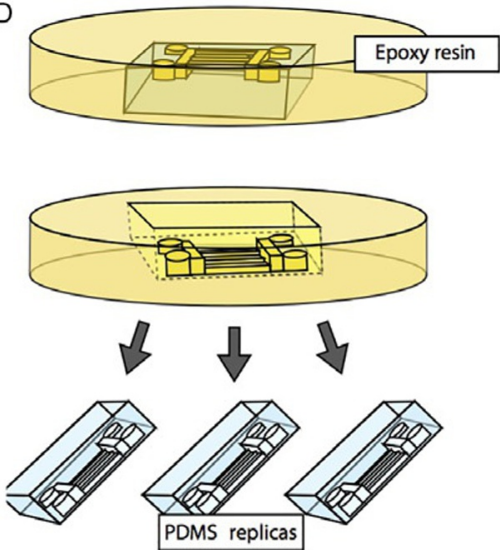

$\mathrm{E}$
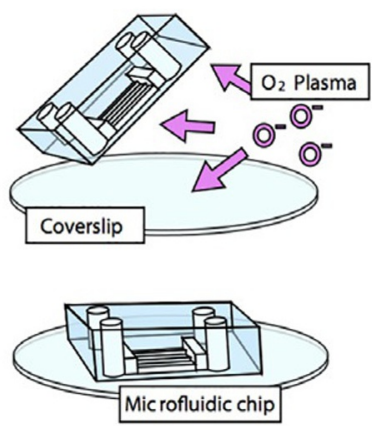

$\mathrm{F}$
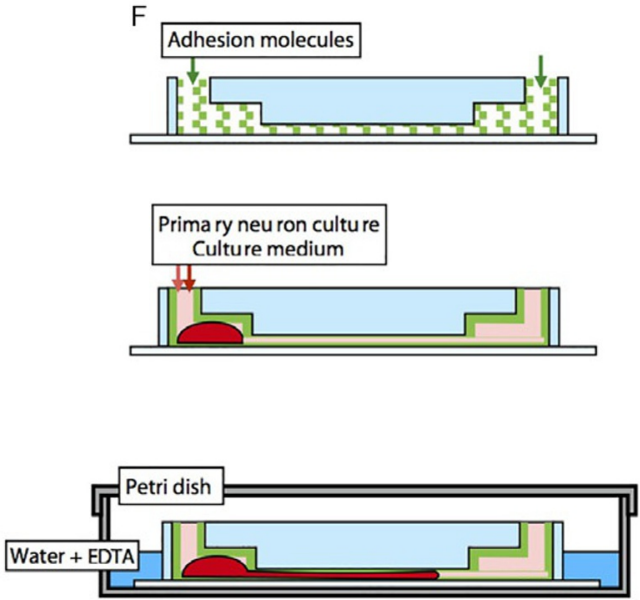

FIG. 4

Microfluidic chips fabrication protocol. (A) Microchannels photolithography: spin-coating of photoresist, insolation and development. (B) Culture chambers photolithography and fabrication of the master. (C) PDMS reticulation on master and fabrication of a PDMS replica. (D) Resin mold preparation from replica and chips preparation from the mold. (E) Bonding of PDMS replica and glass coverslip. (F) Preparation of the microfluidic chip for culture, seeding of neurons and culture conditions. 
11. Immerse the wafer for $1 \mathrm{~min}$ in $50 \mathrm{~mL}$ of developer contained into a $10 \mathrm{~cm}$ diameter beaker shaked at $90 \mathrm{rpm}$ on a rotary shaker.

12. Remove the wafer from the developer bath and place it above the "trash" dish. Briefly sprinkle the wafer for a few seconds with developer, then with isopropanol.

13. Place the wafer on an absorbent paper and dry it with pressurized nitrogen using an air blow gun.

14. Hard bake: place the wafer on a hotplate regulated at $200^{\circ} \mathrm{C}$ for about $5-10 \mathrm{~min}$ to eliminate surface cracks.

15. Check the quality of the structures under an optical microscope.

16. Check the height of the microchannels with a profilometer.

\subsection{Second layer (see Fig. 4B)}

1. Cut thin stripes of adhesive tape. Cover the alignment crosses on the first photoresist layer with these stripes. Be careful not to overlay the surface where chambers will be imprinted. A part of these stripes should protrude on the sides of the wafer for easy removal.

2. Place the wafer on a spin-coater. Check that the spin-coater is working properly by verifying that the aspiration of the substrate is effective (the substrate should spin and stay in place during the nominal rotation speed).

3. Pour a volume of SU8-2050 to cover about $4 \mathrm{~cm}$ in diameter of the wafer on its center. Do it directly from the container, due to the high viscosity of SU8-2050.

4. Spin-coat the wafer with the resin in two steps:

a. $500 \mathrm{rpm}$ for $10 \mathrm{~s}$ with acceleration of $100 \mathrm{rpm} / \mathrm{s}$.

b. $3000 \mathrm{rpm}$ for $30 \mathrm{~s}$ with acceleration of $300 \mathrm{rpm} / \mathrm{s}$.

5. Gently remove the adhesive tape.

6. Soft bake: place the coated wafer on a hotplate regulated at $65^{\circ} \mathrm{C}$ for $2 \mathrm{~min}$, then on a second one regulated at $95^{\circ} \mathrm{C}$ for $7 \mathrm{~min}$. Place the wafer on the bench and let it cool down at room temperature.

7. Place a transparent glass plate on the mask holder of the mask aligner and turn on the vacuum to secure it. Insert the holder into the mask aligner slide channels. The plastic mask encoding the geometry of the culture chambers will be glued later by capillarity to this transparent support.

8. Place the plastic mask on the silicon wafer, ink side facing the photoresist, and roughly align the alignment crosses by hand.

9. Place the silicon wafer and plastic mask on the chuck of the mask aligner.

10. Put a small drop of distilled water (around $50 \mu \mathrm{L}$ ) on top of the plastic mask and slide the chuck in working position. Do not deposit too much water, or it will spill through the sides of the plastic mask and perturb the alignment step.

11. Lift up the chuck until achieving hard contact. The plastic mask will stick by capillarity to the glass plate, while remaining roughly aligned with the silicon wafer. 
12. Activate the separation lever of the mask aligner. This allows a slight lowering of the wafer and consequently hard contact suppression for easy alignment.

13. Move the chuck with the micrometric screws of the aligner to superpose the alignment marks of the two layers: first by adjusting the angle between them, then by changing $x / y$ position. The mask should not move during this operation.

14. Put the separation lever back to its initial position (i.e., come back to hard contact).

15. Expose the second layer through the mask. Exposure dose should be $170 \mathrm{~mJ} / \mathrm{cm}^{2}$. For calculating the exposure time, take into account the absorbance of the plastic: around $20 \%$ at $385 \mathrm{~nm}$.

16. Remove the wafer from the aligner and soft bake as in step 6. Ensure that the wafer has cooled down at room temperature by waiting 1-2 min at the end of this step.

17. Repeat steps $11-13$ of Section 2.2.2.2.1 using now a soaking time in the developer of $6 \mathrm{~min}$.

18. Check the quality of the structures under an optical microscope.

19. Check their height with a profilometer.

\subsubsection{Mold production (see Fig. 4C and D)}

SU8 resin molds are not suitable for mass production of chips: microchannels can detach and the silicon wafer is easily breakable. The production of an epoxy resin mold from the initial PDMS replicas allows a virtually infinite production of chips while retaining fine details.

1. Pour $45 \mathrm{~g}$ of silicon-based organic polymer (PDMS) into a $250 \mathrm{~mL}$ plastic cup. Add $5 \mathrm{~g}$ of its curing agent (1:10 in weight). Stir the mixture using a plastic pipette for $2-3 \mathrm{~min}$ until achieving high bubble content, indicating adequate mixing.

2. Place the mixture within a vacuum desiccator and pump until all air bubbles trapped into the PDMS are eliminated. Watch over the desiccator during vacuum generation: to avoid foam overflow, tap it on the lab bench to break bubbles, or break the vacuum during degassing.

3. Place the master mold in a square $10 \mathrm{~cm}$ wide plastic dish and pour the mixture on the mold, close to the surface of the wafer.

4. Place the PDMS-mold device in the oven set at $70^{\circ} \mathrm{C}$ for at least $3 \mathrm{~h}$.

5. Demold the PDMS block, by introducing a plastic micropipette tip between the side of the dish and the PDMS block, and rotating it around the PDMS block several times. When the PDMS block still attached to the wafer can be held, remove both of them carefully from the dish. This step needs to be performed with caution, as the silicon wafer is very brittle.

6. Cut around the silicon wafer and demold the PDMS replica using a scalpel.

7. Cut around (leave a $2 \mathrm{~mm}$ margin from future inlets positions) the chip using a scalpel or a razor blade. 
8. Repeat steps 1-7 to get a number of PDMS replicas for the production of a big resin mold encoding several chips.

9. Cut adhesive double-sided tape stripes whose size is a little bigger than the PDMS chips. Stick these stripes on the bottom of a silicone mold (cake mold), while keeping a minimum spacing between them $(1 \mathrm{~cm})$ and with the silicone mold edges $(2 \mathrm{~cm})$.

10. Stick the PDMS chips on these adhesive stripes, circuit side up. Pay attention that the edges of the chips are correctly stuck to the tape, or epoxy resin will infiltrate under the chips.

All steps involving epoxy resin before the $150^{\circ} \mathrm{C}$ baking step, including baking itself, should be performed under a fume hood as the vapors are toxic.

11. Pour a volume of epoxy resin base in a disposable plastic cup. Count $250 \mathrm{~mL}$ of resin for a $20 \mathrm{~cm}$ wide mold.

12. With a pipetboy, introduce $2 \%(\mathrm{v} / \mathrm{v})$ of the epoxy resin catalyst in the beaker. It is important to avoid making bubbles as much as possible when homogenizing the mix, as they might break the resin during baking. Trick: inject the catalyst in the resin with the slower mode of the pipetboy while moving the pipet in the liquid resin. Then remove the pipet from the pipetboy and slowly mix following an "8" shape alternatively with circles.

13. Pour the mixed resin into the silicone mold containing the PDMS chips: be quick and maintain the beaker close to the surface to prevent the formation of bubbles.

14. Put the silicone mold in an oven located under a fume hood, set to $30^{\circ} \mathrm{C}$.

15. Wait $3 \mathrm{~h}$ for the resin to polymerize, turn off the oven while letting it closed and let it cool down to room temperature under the fume hood. The polymerization step is strongly exothermic. Avoid brutal temperature changes as they may crack the resin.

16. Remove the epoxy resin from the silicone mold, and the PDMS block and the tape from the resin.

17. Wrap your resin mold into aluminum foil to homogenize temperature changes during the subsequent heating and cooling steps.

18. Place the aluminum-wrapped resin in the same oven and bake at $150^{\circ} \mathrm{C}$ overnight in order to evaporate toxic compounds from the resin.

19. Turn off the oven while letting the door closed for progressive cooling down of the resin to room temperature. This step might take several hours.

20. Remove the aluminum foil.

\subsubsection{Chamber fabrication (see Fig. 4E)}

Note: the first two batches of PDMS made with the resin mold will not be suitable for cell culture due to residues of toxic compounds.

1. Repeat steps 1-7 of Section 2.2.2.2 (PDMS replicas production). The silicon/ photoresist mold is now replaced by the resin mold. 
2. Create inlets by pressing the $4 \mathrm{~mm}$ diameter biopsy puncher firmly and actuate it to cut and carve openings at both extremities of the culture chambers. The circular structures at the extremities of the culture chambers indicate the positions at which inlets should be generated.

3. Clean the chip by sticking and peeling adhesive tape on the microstructured side. Sprinkle isopropanol on both sides. Then dry the chip with pressurized nitrogen using an air blow gun.

4. Clean the glass coverslips with a dust-free wipe imbibed with isopropanol, then spray them with pressurized nitrogen using an air blow gun.

5. Treat the glass coverslips and the chips, microchannels side up, in the plasma cleaner. Plasma parameters for a Diener Pico PCCE: pumping down pressure: 0.25 mbar; $\mathrm{O}_{2}$ supply duration: $3 \mathrm{~min}$; gas flow: $10 \mathrm{sccm}$; maximum deviation: $\pm 5 \mathrm{sccm}$; plasma duration: $30 \mathrm{~s}$; set pressure: 0.40 mbar; maximum deviation: \pm 0.10 mbar; set power: $50 \mathrm{~W}$; maximum deviation: $5 \%$; venting duration: $45 \mathrm{~s}$.

6. Immediately after plasma treatment, gently put the activated PDMS chip in contact with the glass coverslip, and delicately apply pressure on the chip to bond it to the coverslip. No unbonded regions should subsist between the inlets and the side of the chips, or between distinct inlets.

7. Under the hood and within $30 \mathrm{~min}$ after bonding, fill one inlet by chamber with distilled water. This prevents the PDMS from reverting to its original hydrophobic state.

8. Put the assembled chips in petri dishes.

\subsubsection{Chamber preparation: Day 1 (see Fig. 4F)}

1. Place the chips and the petri dishes, open with the inside of the lid facing up, under UV light for $20 \mathrm{~min}$ for sterilization.

All steps following UV sterilization should be performed under a cell culture hood with sterile equipment and solutions. To inject liquid into a PDMS chip, place a $1 \mathrm{~mL}$ tip cone filled with liquid just above the inlet, then press the pipette to deposit a single drop at the entrance of the inlet. Emptying an inlet can be performed either with an electric pump or a $1 \mathrm{~mL}$ micropipette, and should be done with a lot of care after cells have been seeded in the chips.

2. Fill with a $100 \mu \mathrm{L}$ pipette cone $30 \mu \mathrm{L}$ of a $10 \mu \mathrm{g} / \mathrm{mL}$ PDL solution in PBS in one inlet per culture chamber. Inject in diametrically opposed inlets (i.e., located on each side of the microchannels). This allows the PDL solution to flow through the microchannels.

3. Incubate overnight in the cell culture incubator regulated at $37^{\circ} \mathrm{C}$ and $5 \%$ of $\mathrm{CO}_{2}$. Humidified atmosphere limits evaporation.

\subsubsection{Cells injection and culture maintenance: From day 0 (see Fig. 4F)}

1. Before your dissection protocol, empty the PDL solution from your chips. Introduce a solution of $2.5 \mu \mathrm{g} / \mathrm{mL}$ laminin in PBS, following the same filling process as with the PDL solution. Incubation should last at least $4 \mathrm{~h}$ in the 
cell culture incubator. The flow of laminin solution through the chambers serves as a washing step for PDL. If no laminin is used, perform the same step with PBS.

2. Prepare your dissociated neurons suspension. The protocol used for dissection and dissociation, and the composition of the culture medium can be found in Tan et al. (2018).

3. Resuspend your neurons at a high concentration in culture medium, here 40 millions cells $/ \mathrm{mL}$, using a $200 \mu \mathrm{L}$ tip cone.

4. Empty the laminin from the chips.

5. Introduce a drop of medium in the two inlets of the culture chamber opposite to the one in which you will introduce the cells (i.e., located on the other side of the microchannels). This prevents the cells from accumulating at the entrance of the microchannels in the cell seeding chamber or even pass through them due to overpressure during the seeding step.

6. Inject $1.5 \mu \mathrm{L}$ of cell suspension at a single inlet of the cell seeding chamber with a $10 \mu \mathrm{L}$ tip.

7. Leave cells to adhere from 5 to 20 min before adding an equal volume of warm culture medium in each inlet.

8. Add $3 \mathrm{~mL}$ of a distilled water $+0.5 \%$ EDTA solution in the petri dish, around your chip. This helps preventing evaporation. EDTA prevents contamination of the water. Be careful not to cover your chip, as the water+ EDTA solution would flow into your culture chambers through the inlets.

9. Put your chips in the cell culture incubator.

10. Half of the cell culture medium should be changed at day in vitro (DIV) 6 and 13 for primary murine neurons. Water+EDTA level should be maintained during culture duration. If using an electric pump, be careful not to introduce the nozzle to the bottom of the inlet as you may pump the cells out of the chamber.

\subsection{IMAGING: ACQUISITION AND DISPLAY}

Fluorescence imaging was performed at DIV 11 using a $20 \times$ magnification. For an efficient automated image analysis, each set of microchannels should be positioned almost identically in the field of view (e.g., be careful to keep a similar orientation of the microchannels between successive images). For repeated imaging (videomicroscopy) of live neuronal cultures, be careful to limit laser exposure time to the minimum, as this can be detrimental to neuronal survival. Several $Z$ plans (Z-stack) can be acquired for each position, to account for eventual inclination of the sample.

\subsection{AUTOMATED IMAGE ANALYSIS}

In the following, the term "structure" represents the microchannels design of a given device. The automated image analysis solution presented here (see Supplementary Material "code_arches_analysis") primarily relies on a template matching algorithm to extract from a large number of widefield phase contrast images structures 
specified manually as templates beforehand by the user. The template matching function first applies a sobel edge detection filter on both the image to analyze and the template, bins both the image and the template to increase processing speed, then generates a convoluted image using the template as the convolution kernel. The positions and intensities of peaks in the convoluted image indicate the position and "quality" of the matches. The script automatically assess what structures are present in each image, their locations and orientations by varying the template and the orientation of the image and looking at the matches quality. This alleviates the need to properly label all the raw images. However, it is primordial that (1) the microstructures from a same type are fabricated in a reproducible fashion and (2) microstructures of different types exhibit significant visual differences, since the template matching function solely relies on geometrical similarity.

1. Provide a phase contrast (phase) template for each structure for which axonal filtration has to be quantified. A script comprising a graphical user interface makes that task easier.

2. The raw images to be analyzed (Z-stacks) are projected using the max intensity value across the different sections.

3. The orientation and structure type of each projected image are determined by finding the template and angle that yield the best match. The phase and fluorescence images are then aligned based on this orientation.

4. The algorithm determines the positions of the different matches (repeated pattern: here two series of arches + straight microchannels) on the picture, and saves each phase contrast and fluorescence patch to a separate file. Those files are coarsely aligned due to the binning step in the template matching function.

5. All the phase patches for a given structure are loaded into a stack, and realigned with single pixel precision. The shift values are used to align in turn the fluorescence stacks. The direction of axonal growth (forward or reverse) is determined based on the fluorescence intensity in each culture chamber, the side with the cell bodies being much brighter.

6. The transmission values for each image in the stack are calculated as $T=(A-B) /(S-B)$ where $A$ is the fluorescence intensity in the axonal compartment, $S$ in the Soma compartment, and $B$ in a background area devoid of processes.

7. The selectivity for each structure is calculated as the average $\left(T_{\text {forward }}\right) /$ average $\left(T_{\text {reverse }}\right)$.

\section{RESULTS}

In this work, we sought to recreate oriented neuronal networks in vitro through the use of asymmetric microchannels relying on the "arches" design, as initially described in Renault et al. (2016). 
To direct axonal growth, we designed three different microchannels arrays. The first one contains $10 \mu \mathrm{m}$ wide straight microchannels linked by $100 \mu \mathrm{m}$ diameter, $10 \mu \mathrm{m}$ wide arches (Fig. 2D). 6 and 7 repeats of these arches were drawn on every pair of microchannels, but 2 are buried under the central chamber during microfabrication, thus reducing to $4 / 5$ the number of arches. Therefore, circuits containing this design will be referred to as "arches 100 4R." Another variant relies on the presence of $40 \mu \mathrm{m}$ instead of $100 \mu \mathrm{m}$ diameter arches (Fig. 2E), and will be referred to as "arches 40 4R." The last variant was designed with 9 repeats of $100 \mu \mathrm{m}$ diameter arches and a greater proximity of the cusps formed by those arches in an effort to maximize their number (Fig. 2F). A central space was managed for the central chamber. These will be called "sawtooth arches" in reference to the frequent repetition of pointed cusps along the microchannels. In each design, the "forward," permissive direction of axonal growth goes from left to right. The non-permissive one will be referred as "reverse." Each of them contains opposed funnels and traps adjacent to the culture chambers for respectively harvesting and filtering a maximum number of neuronal processes before microchannel openings.

We evaluated the axonal transmission of these different designs in the "forward" (neurons seeded in the "emitting" chamber) and "reverse" directions (neurons seeded in the "receiving" chamber). To this end, primary murine cortical $(\mathrm{Cx})$ or hippocampal neurons (Hip) extracted from a tdTomato-expressing mouse line were seeded in the emitting or receiving culture chambers, as pictured in Fig. 4F, and left to grow for 11 days before being imaged with phase contrast and fluorescence microscopy. Image analysis is a laborious task when performed manually. We thus aimed to develop an automatized solution achieving a visual estimation of the average density of axons in the forward and reverse directions and to obtain a preliminary numerical estimation of the transmission coefficients. This technique, already used in Renault et al. (2016), is described in Section 2. This process allowed for the fast analysis of a consequent amount of pictures with minimal human input, and the pre-selection of designs with the most promising features. Fig. 5 presents the results of this analysis, with average axonal paths pictured in Fig. 5A. The selectivity values obtained from the ratio between forward and reverse transmission coefficients generated by the script were 3.34 for the " $1004 \mathrm{R}$ " design, 1.75 for " 40 4R" and 15.67 for sawtooth arches seeded with Cx. A 6.56 transmission ratio value was calculated in the sawtooth arches seeded with hippocampal neurons (Fig. 5D). A closer examination of these pre-screening results revealed that the differences in the transmission ratio were not due to the forward transmission value in the designs seeded with $\mathrm{Cx}$, which were similar in the three designs (Fig. 5B), but to differences in reverse transmission values. These values were extremely decreased in sawtooth arches, mildly reduced in arches $1004 \mathrm{R}$ and still elevated in arches 40 4R (Fig. 5C). The transmission ratio obtained for Hip in sawtooth arches was the result of a weaker axonal transmission in the forward direction compared to $\mathrm{Cx}$, while reverse growth was still extremely weak in the reverse direction. These preliminary results allowed for classifying our designs according to their filtration power, and indicated that sawtooth arches had the best performances. 
18 Microchannels for directed axonal growth

A

$1004 R$

Cortex

Forward

$1004 R$

Cortex

Backward

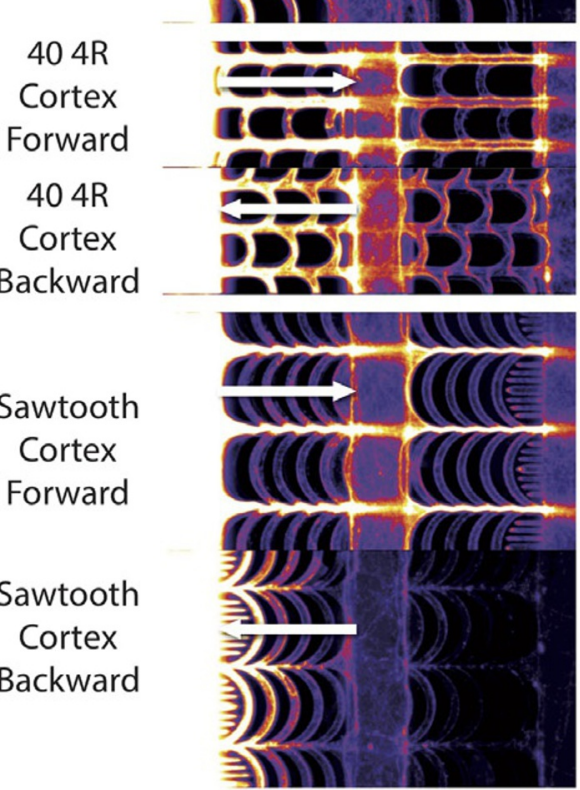

Sawtooth

Hipp.

Forward

Sawtooth

Hipp.

Backward
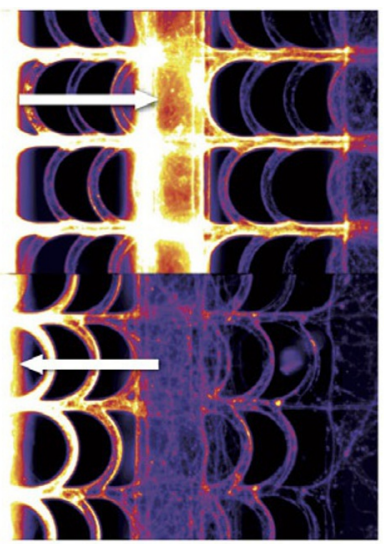
Although this automatic method allowed for easy and fast analysis of the pictures and for the classification of the designs based on their filtration efficiency, it did not completely reflect the reality, in particular in the cases where the reverse transmission is very low, i.e., when the process of background subtraction is of critical importance. Indeed, a closer examination of individual pictures (Fig. 6A) revealed that reverse axonal outgrowth was quasi nonexistent in sawtooth arches, with an average of 0.12 axons per microchannel growing in the reverse direction for $\mathrm{Cx}$ and no axon at all when hippocampal neurons were seeded. In comparison, an average of 1.48 and 8.02 axons per microchannel crossed the " $1004 \mathrm{R}$ " and " $404 \mathrm{R}$ " arches in the reverse direction, respectively (Fig. 6C). Axonal outgrowth from cortical neurons in the forward direction was extremely robust in the three structures, with an average 24.16, 16.59 and 20.4 axons per microchannel for the "100 4R," "40 4R" and sawtooth arches, respectively. Hippocampal forward axonal outgrowth in sawtooth arches was quite weak, with 4.28 axons per microchannel (Fig. 6B). Calculation of the transmission ratios from these manually acquired values showed that sawtooth arches allowed a selectivity of $339(99.7 \%)(n=15$ cultures in 3 separate experiments, with 10 microchannels per replicate). This value was infinite (100\%) for hippocampal cultures, as no axon crossed in the reverse direction ( $n=7$ cultures in 2 separate experiments, with 10 microchannels per replicate). Arches " $1004 \mathrm{R}$ " and " $404 \mathrm{R}$ " had respective selectivity of 16.3 (93.9\%) and 2.07 (51.7\%) (Fig. 6D). To our knowledge, the sawtooth arches present the best axonal filtration ratios described in the literature for this level of compactness and innervation density.

The next step was to assess how sawtooth arches fare in a network situation. To this end, we seeded the emitting chamber of the circuit with wild-type cortical neurons, while tdTomato-expressing hippocampal neurons were introduced in the receiving compartment. Cultures were imaged after 11 days of culture (Fig. 7). We observed minimal hippocampal outgrowth to the emitting chamber (Fig. 7B). Filtration of hippocampal neurons was thus similar when neurons were seeded in only one chamber or both, with an average of respectively 0 (Fig. 6C) and 0.05 axons

\section{FIG. 5}

Automatic preliminary screening of axonal growth through asymmetric microchannels. Neurons extracted from a tdTomato-expressing mouse line were either seeded in the "emitting" ("Forward" situation) or "receiving" ("Backward" situation) culture chambers and left to grow for 11 days. Signal corresponds to tdTomato fluorescence. (A) Cortical ("Cortex") neurons were seeded on either side of culture devices containing $100 \mu \mathrm{m}$ arches with 4 repeats ("100 4R"), $40 \mu \mathrm{m}$ arches with 4 repeats ("40 4R") or "sawtooth" arches.

Hippocampal ("Hipp.") neurons were in addition seeded on either side of "sawtooth" arches. Scale bar is $200 \mu \mathrm{m}$. (B) Quantification of the average transmission (signal intensity in the axonal chamber normalized by signal intensity in the somatic chamber, both minus background signal) in the "forward" direction, and $(\mathrm{C})$ in the "reverse" direction. Error bars correspond to $95 \%$ confidence interval $\left(* * p<0.01,{ }^{* * *} p<0.001,{ }^{* * * *} p<0.0001\right)$. $N=$ minimum two independent experiments. (D) Ratio of the average "forward" on "reverse" transmissions. 
20 Microchannels for directed axonal growth

A

$1004 \mathrm{R}$
Cortex

Forward

$1004 R$

Cortex

Backward

$404 \mathrm{R}$

Cortex

Forward

$404 R$

Cortex

Backward

Sawtooth

Cortex

Forward

Sawtooth

Cortex

Backward

(
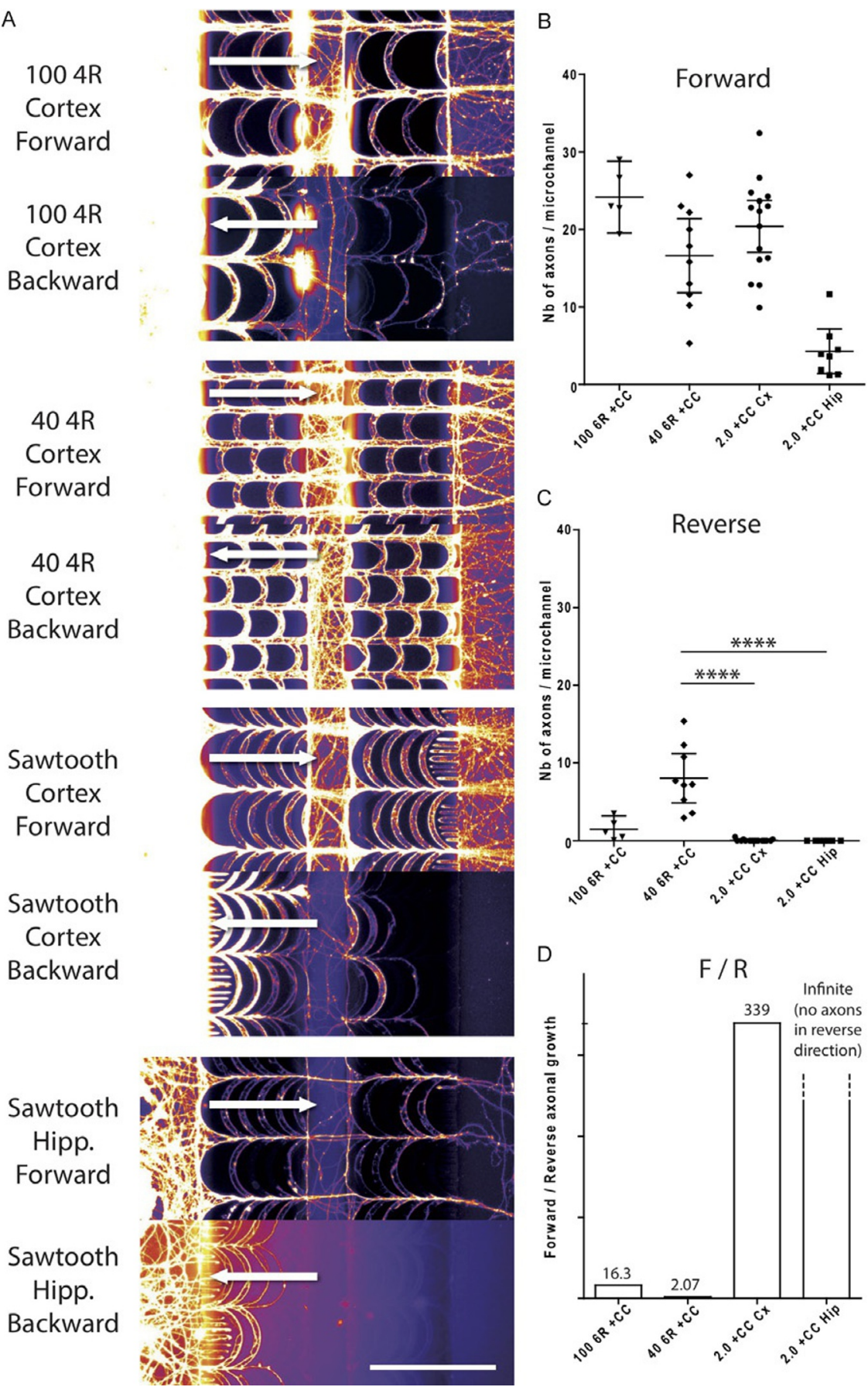

FIG. 6

See legend on opposite page. 
growing in the reverse direction (Fig. 7C). This supports the notion that our design allows for the reconstruction of extremely directed neuronal networks in vitro.

\section{DISCUSSION}

We have described in this methodological work several microchannels designs relying on the "arches" paradigm reported in Renault et al. (2016) and evaluated their filtration power first through an automatic pre-screening technique and then through quantification at the single axon level. All these designs aimed at filtering axonal growth in a unidirectional fashion between two neuronal cultures. Importantly, the "sawtooth" channels described here exhibited very good filtration characteristics. We obtained a filtration ratio at DIV 11 of $99.7 \%$ for cortical neurons and of $100 \%$ for hippocampal neurons. Importantly, this characteristic was preserved in a network situation where both chambers were seeded. To our knowledge, we describe yet the most efficient axonal filtration system through the use of short and compactly interspaced microchannels.

Sawtooth arches exhibited a very high axonal filtration in the reverse direction, significantly higher than the two other designs. This might be linked to the higher number of arches, 9 instead of 4 per straight microchannel. Indeed Renault et al. (2016) described a progressive filtration of axons at each arches repeat, thus supporting an additive property (although non-linear) of arches succession. Another factor to take into account was the closer proximity of successive cusps and their increased acuteness as compared to $100 \mu \mathrm{m}$ diameter arches. This characteristic might result in a more severe filtration of reverse axonal growth.

Of great interest, sawtooth arches were able to constrain reverse axonal growth in a network situation, while the design of $100 \mu \mathrm{m}$ diameter arches reported in Renault et al. (2016) displayed reduced filtration capacities in a similar context. This might be due to an earlier filtration of reverse axonal growth, thus minimizing the probability

\section{FIG. 6}

Axonal growth through asymmetric microchannels. Neurons extracted from a tdTomatoexpressing mouse line were either seeded in the "emitting" "Forward" situation) or "receiving" ("Backward" situation) culture chambers and left to grow for 11 days. Signal corresponds to tdTomato fluorescence. (A) Cortical ("Cortex") neurons were seeded on either side of culture devices containing arches $100 \mu \mathrm{m} 4$ repeats ("100 4R"), arches $40 \mu \mathrm{m} 4$ repeats ("40 4R") or "sawtooth" arches. Hippocampal ("Hipp.") neurons were seeded on either side of "sawtooth" arches. Scale bar is $200 \mu \mathrm{m}$. (B) Quantification of the number of axons per microchannel reaching the other chamber in the "forward" direction, and $(\mathrm{C})$ in the "backward" direction. Error bars correspond to $95 \%$ confidence interval $(* * * * p<0.0001) . N=$ minimum two independent experiments. (D) Ratio of the average "forward" on "backward" axon growth. 


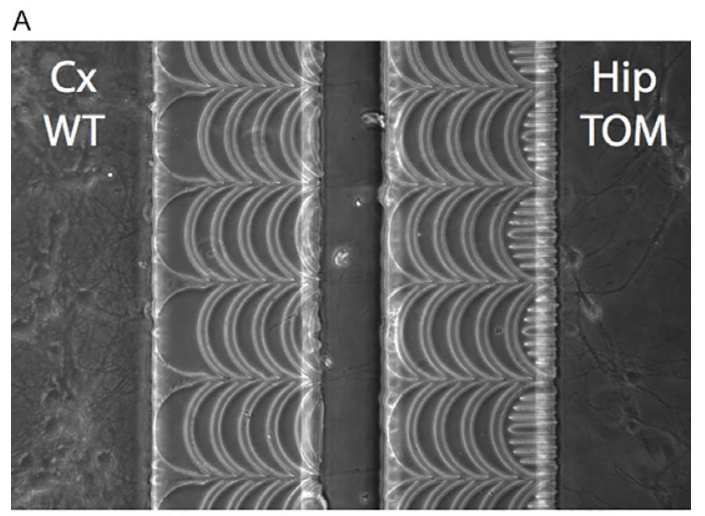

B

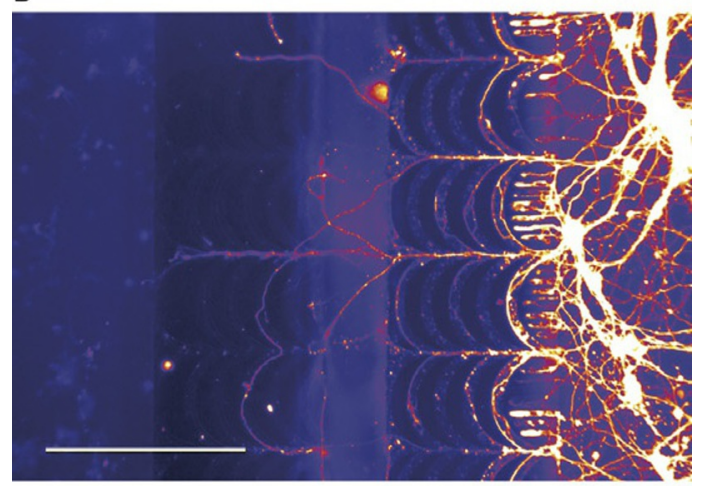

C

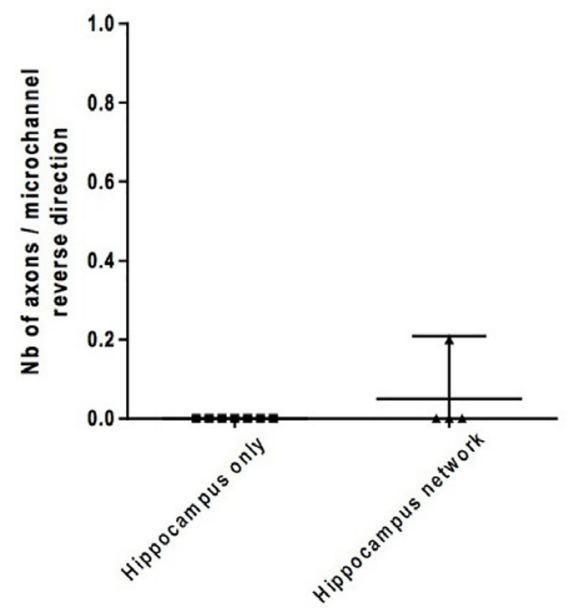

FIG. 7

Minimal retrograde axonal growth in cortico-hippocampal networks. Hippocampal neurons extracted from a tdTomato-expressing mouse line were seeded in the "receiving" culture chamber of a "sawtooth" arches microfluidic chips while cortical neurons from a wild-type mouse line were seeded in the "emitting" culture chamber. (A) Phase contrast and (B) tdTomato fluorescence. Scale bar is $200 \mu \mathrm{m}$. (C) Quantification of the number of hippocampal axons by microchannels growing in the reverse direction at DIV 11, with only TOM hippocampal neurons being seeded in the receiving chamber, or with WT cortical neurons ( $\mathrm{Cx}$ ) seeded in the emitting chamber and TOM hippocampus (Hip TOM) seeded in the receiving one. Error bars correspond to 95\% confidence interval. 
that axons growing in the reverse direction would be guided by axons traveling in the forward direction through a fasciculation phenomenon.

The filtration of hippocampal and cortical axons differed in our design, and can be attributed to the differences between the fasciculation properties of these two neuronal subtypes. Indeed, we observed (Fig. 5A) that cortical axons remained bundled in the central channel whereas the bundles formed by hippocampal neurons inside the microchannels tend to defasciculate right at their exit, leading to a decreased forward transmission compared to cortical neurons. This observation supports the notion that axons of each neuronal subtype has unique axonal navigation parameters. These features should be taken into account when planning on reconstructing neuronal networks, and filtration efficiency should be evaluated for each new subtype before doing so.

The automatic image analysis script we presented here allowed for fast and easy display of a vast amount of images, and to a preliminary classification in terms of transmission and axonal selectivity. However, the transmission ratios generated by this method are quite underestimated due to the sensitivity of this method to the value of the background signal. Altogether, this solution still represents a convenient process for pre-screening a vast number of structures before precisely quantifying the most promising ones.

The arche-based microchannels designs presents several advantages over a funnel-shaped design, including less severe mechanical constraints as already discussed in Renault et al. (2016). We provide here a methodology to design and fabricate variants of arche-based devices to achieve selectivity above $99.7 \%$. Interestingly, a filtration efficiency of $100 \%$, although not demonstrated in a network setting, was described in $\mathrm{Na}$ et al. (2016). This method relied on axonal "memory" of previous path, which meant that the longer an axon follows a straight path in a microchannel, the higher the probability it will not deviate from this path if it encounters an alternative route. One of the disadvantages presented by this device is the low density of microchannels, with one microchannel every $500 \mu \mathrm{m}, 5$ times lower than for sawtooth arches. This characteristic might impede the reconstruction of a functional network. Moreover, the total microchannel length needed for efficient filtration was $1.5 \mathrm{~mm},>3$ times longer than for our device, in which the microchannels are $400 \mu \mathrm{m}$ long.

Arche-based microchannel designs open the way for the reconstruction of physiologically relevant models for the study of pathological processes occurring in neuronal networks. Of particular importance is the possibility to build composite networks with various neuronal subtypes and mutant neurons. The independent access to each of the neuronal compartments permits the individual stimulation, inhibition, treatment, transfection and observation of each of the network nodes. One of the main research areas that could benefit from this technological achievement is the study of the trans-neuronal transfer of pathological agents. Indeed, the study of the propagation of prion-like phenomena-involved in Alzheimer's and Parkinson's diseases among others - in neuronal networks can currently only be performed with bidirectional systems (Mao et al., 2016; Tran et al., 2014) or with hybrid co-cultures 
(Calafate et al., 2015). As the directionality of this propagation is still the subject of debates in the scientific community (Uchihara \& Giasson, 2016), being able to address this question directly in extremely directed networks would improve our comprehension of the etiology of these diseases.

\section{ACKNOWLEDGMENT}

Part of this work was supported by the European Research Council Advanced Grant Number 321107 "CellO".

\section{REFERENCES}

Calafate, S., Buist, A., Miskiewicz, K., Vijayan, V., Daneels, G., de Strooper, B., et al. (2015). Synaptic contacts enhance cell-to-cell tau pathology propagation. Cell Reports, 11(8), 1176-1183.

Campenot, R. B. (1977). Local control of neurite development by nerve growth factor. Proceedings of the National Academy of Sciences of the United States of America, 74(10), 4516-4519.

Chubakov, A. R., \& Sotnikov, O. S. (1982). Formation of connections between the raphe and hippocampal nuclei in tissue culture (intravital functional and morphological studies). Arkhiv Anatomii, Gistologii i Émbriologii, 82(3), 11-20.

Corey, J. M., Wheeler, B. C., \& Brewer, G. J. (1991). Compliance of hippocampal neurons to patterned substrate networks. Journal of Neuroscience Research, 30(2), 300-307.

Deleglise, B., Lassus, B., Soubeyre, V., Alleaume-Butaux, A., Hjorth, J. J., Vignes, M., et al. (2013). Synapto-protective drugs evaluation in reconstructed neuronal network. PLoS One, 8(8), e71103.

Deleglise, B., Magnifico, S., Duplus, E., Vaur, P., Soubeyre, V., Belle, M., et al. (2014). $\beta$-Amyloid induces a dying-back process and remote trans-synaptic alterations in a microfluidic-based reconstructed neuronal network. Acta Neuropathologica Communications, 2, 145.

Gladkov, A., Pigareva, Y., Kutyina, D., Kolpakov, V., Bukatin, A., Mukhina, I., et al. (2017). Design of cultured neuron networks in vitro with predefined connectivity using asymmetric microfluidic channels. Scientific Reports, 7(1), 15625.

Kale, P., Zalesky, A., \& Gollo, L. (2018). Estimating the impact of structural directionality: How reliable are undirected connectomes? Network Neuroscience, 1, 1-51.

Le Feber, J., Postma, W., De Weerd, E., Weusthof, M., \& Rutten, W. L. (2015). Barbed channels enhance unidirectional connectivity between neuronal networks cultured on multi electrode arrays. Frontiers in Neuroscience, 9, 412.

Mao, X., Ou, M. T., Karuppagounder, S. S., Kam, T. I., Yin, X., Xiong, Y., et al. (2016). Pathological $\alpha$-synuclein transmission initiated by binding lymphocyte-activation gene 3 . Science, 353(6307), aah3374.

Na, S., Kang, M., Bang, S., Park, D., Kim, J., Sim, S. J., et al. (2016). Microfluidic neural axon diode. Technology, 04, 240-248.

Peyrin, J. M., Deleglise, B., Saias, L., Vignes, M., Gougis, P., Magnifico, S., et al. (2011). Axon diodes for the reconstruction of oriented neuronal networks in microfluidic chambers. Lab on a Chip, 11(21), 3663-3673. 
Renault, R., Durand, J. B., Viovy, J. L., \& Villard, C. (2016). Asymmetric axonal edge guidance: A new paradigm for building oriented neuronal networks. Lab on a Chip, 16(12), 2188-2191.

Renault, R., Sukenik, N., Descroix, S., Malaquin, L., Viovy, J. L., Peyrin, J. M., et al. (2015). Combining microfluidics, optogenetics and calcium imaging to study neuronal communication in vitro. PLoS One, 10(4), e0120680.

Tan, V. X., Lassus, B., Lim, C. K., Tixador, P., Courte, J., Bessede, A., et al. (2018). Neurotoxicity of the cyanotoxin BMAA through axonal degeneration and intercellular spreading. Neurotoxicity Research, 33(1), 62-75.

Taylor, A. M., Blurton-Jones, M., Rhee, S. W., Cribbs, D. H., Cotman, C. W., \& Jeon, N. L. (2005). A microfluidic culture platform for CNS axonal injury, regeneration and transport. Nature Methods, 2(8), 599-605.

Taylor, A. M., Dieterich, D. C., Ito, H. T., Kim, S. A., \& Schuman, E. M. (2010). Microfluidic local perfusion chambers for the visualization and manipulation of synapses. Neuron, 66(1), 57-68.

Tran, H. T., Chung, C. H., Iba, M., Zhang, B., Trojanowski, J. Q., Luk, K. C., et al. (2014). Alpha-synuclein immunotherapy blocks uptake and templated propagation of misfolded alpha-synuclein and neurodegeneration. Cell Reports, 7(6), 2054-2065.

Uchihara, T., \& Giasson, B. I. (2016). Propagation of alpha-synuclein pathology: Hypotheses, discoveries, and yet unresolved questions from experimental and human brain studies. Acta Neuropathologica, 131(1), 49-73.

Virlogeux, A., Moutaux, E., Christaller, W., Genoux, A., Bruyere, J., Fino, E., et al. (2018). Reconstituting corticostriatal network on-a-chip reveals the contribution of the presynaptic compartment to Huntington's disease. Cell Reports, 22(1), 110-122.

Volpicelli-Daley, L. A., Luk, K. C., \& Lee, V. M. (2014). Addition of exogenous alphasynuclein preformed fibrils to primary neuronal cultures to seed recruitment of endogenous alpha-synuclein to Lewy body and Lewy neurite-like aggregates. Nature Protocols, 9(9), 2135-2146.

Wyart, C., Ybert, C., Bourdieu, L., Herr, C., Prinz, C., \& Chatenay, D. (2002). Constrained synaptic connectivity in functional mammalian neuronal networks grown on patterned surfaces. Journal of Neuroscience Methods, 117(2), 123-131. 